\section{El software educativo como estrategia didáctica en matemática}

\author{
Educational software as a didactic strategy in mathematics \\ Jorge Luis Roalcaba-Caro* \\ Juan Pedro Soplapuco-Montalvo*
}

\section{RESUMEN}

La tecnología de información y comunicación constituye un medio esencial en la mejora de las prácticas educativas. EI objetivo de este estudio fue realizar un resumen sobre el uso de software GeoGebra como estrategia didáctica en matemática de estudiantes del nivel secundaria. Se emplea las bases de datos de Google Académico y Scopus publicados en los últimos cinco años. Se obtuvo una muestra total de $2 \mathrm{I}$ artículos científicos. Para la extracción de la información en la revisión se siguió el siguiente procedimiento: Autorías y año de publicación, diseño metodológico de estudio, variables de estudio y conclusiones. Como principal resultado se ha podido observar la necesidad de implementar el software didáctico para que pueda contribuir a la mejora del rendimiento académico de matemática. Se concluye que la utilización del software Geogebra como recurso didáctico permite la mejora del aprendizaje en matemática de los estudiantes de secundaria. Se recomienda utilizar el software educativo en las sesiones de aprendizaje para que los estudiantes logren aprendizajes significativos en matemática.

Palabras clave: Software didáctico, GeoGebra, rendimiento académico, matemática

\footnotetext{
* Doctorando en Educación de la Universidad César Vallejo, Chiclayo, Director I.E. Federico Villarreal, Chiclayo, Correo electrónico: rcaroj@ucvvirtual.edu.pe, ORCID: 0000-0002-2588-I823

* Doctor en Ciencias de la Educación, Maestro en Docencia Universitaria e Investigación Educativa, Licenciado en Educación, por la Universidad Nacional Pedro Ruiz Gallo de Lambayeque, Perú. Docente de la Escuela de Posgrado de la Universidad Cesar Vallejo, Chiclayo. Email: smontalvojp@ucvvirtual.edu.pe, Cel.949570072, ORCID: 0000-0003-463I-8877
}

\section{REVISTATECNOLÓGICA ciencia y educación Edwards Deming}

ISSN: 2600-5867

Atribución/Reconocimiento-NoCo mercial- Compartirlgual 4.0 Licencia Pública Internacional - CC

\section{BY-NC-SA 4.0}

https://creativecommons.org/licenses /by-nc- sa/4.0/legalcode.es

Editado por: Tecnológico Superior Corporativo Edwards Deming

Enero - Julio Vol. 5 - I - 202 I

https://revista-edwardsdeming.com/index.php/es e-ISSN: 2576-097I

Recibido: 04 Marzo 2020

Aprobado: II Diciembre, 2020

Pag 48-56 


\begin{abstract}
Information and communication technology is an essential means in the improvement of educational practices. The objective of this study was to make a summary on the use of GeoGebra software as a didactic strategy in mathematics of high school students. The Google Scholar and Scopus databases published in the last five years are used. A total sample of 21 scientific articles was obtained. To extract the information in the review, the following procedure was followed: Authorship and year of publication, methodological study design, study variables and conclusions. As the main result, it has been observed the need to implement didactic software so that it can contribute to the improvement of academic performance in mathematics. It is concluded that the use of Geogebra software as a didactic resource allows the improvement of learning in mathematics of secondary school students. It is recommended to use educational software in learning sessions for students to achieve meaningful learning in mathematics. Keywords: Didactic software, GeoGebra, academic performance, math
\end{abstract}

\title{
INTRODUCCIÓN
}

En esta investigación trata sobre tanto los estudiantes como los docentes deberían usar el software educativo como una manera de construir su conocimiento como elemento cultural esencial en la sociedad actual.

Jiménez y Jiménez (2017) sostienen que el software matemático GeoGebra es una herramienta adecuada como estrategia didáctica para mejorar la matemática, específicamente la resolución de problemas, en todos los niveles de la educación y de esa manera contribuye a la enseñanza y el logro de aprendizajes significativos en los estudiantes.

Zenteno et al (2020) concluye que al aplicar el software GeoGebra se mejora significativamente el aprendizaje de las medidas de dispersión de los estudiantes y también el rendimiento académico de la matemática de los estudiantes.

Bolaños y Ruíz (20l8) consideran que una propuesta utilizando el GeoGebra, está dentro la innovación docente y consta en la elaboración de un material didáctico, la cual fomenta el desarrollo de las diversas competencias y capacidades matemáticas de los educandos, recopilando actividades planificadas en la sesión de aprendizaje enfocadas al uso de la demostración en el campo de la Geometría. 
La selección del GeoGebra es que une geometría, álgebra y cálculo, que en otros programas trabajan en forma dividida, en este caso en un solo paquete se puede utilizar para enseñar la geometría desde el nivel inicial hasta la universidad, y es un software amigable para la demostración de los teoremas, la cual favorece a la resolución de problemas (Díaz-Nunja et al., 2018).

Una de las clasificaciones de un software educativo es que trabajan como simuladores y en el caso de GeoGebra posee características similares a un simulador, el cual le permite al alumno realizar construcciones alterando los objetos, pero preservando las características originales, de esa manera se convierte en una metodología interactiva apropiada para el desarrollo de tareas significativas de aprendizaje en el área de matemática (Gonzáles et al., 2017).

Guachún y Mora (2018), concluyen que la utilización del GeoGebra nos ayuda a comprender conceptos y permite la interacción entre estudiantes y docentes de tal manera que garantiza un aprendizaje significativo en la matemática. Ramirez et al. (2018), afirma que hay aprendizaje signficativo cuando a los estudiantes se los motiva y se les explica la clase usando herramientas interactivas como el software GeoGebra y de esa manera se profundiza varias maneras de resolver problemas de la vida real.

Mendoza et al. (2018) sostienen que la utilización del software GeoGebra contribuye a mejorar el aprendizaje de matemática en los estudiantes del primer nivel es un recursos interactivo. Sarabia (1995) refiere que la Teoría General de los Sistemas está constituida por procesos y tiene una mirada dinámica de la realidad. Un proceso es todo cambio en el tiempo. Desde esta mirada el objeto, diferenciado y distinguido de su entorno, puedes ser percibido como un proceso y su entorno es constituido por procesos. La Teoría General de los Sistemas, la misma que sustenta la propuesta del modelo de software educativo, que define que los sistemas como conjuntos de elementos que se interrelacionan para lograr un objetivo. El software educativo para su diseño tiene una estructura basada en entrada, proceso y salida, como recurso didáctico. Así mismo como resultado del sistema educativo es el logro de los aprendizajes, la cual implica realización de varias actividades en el proceso y tareas para alcanzar los objetivos.

Siemens (citado por Cueva et al., 2019) afirma que el Conectivismo es una teoría que se basa en la creatividad y la innovación. El aprendizaje como proceso consiste en conectar nodos o base de datos especializados y puede estar fuera del ser humano, en dispositivos informáticos, entendido como conocimiento aplicable.

Según los artículos revisados el nivel de aprendizaje en matemática de los estudiantes de secundaria es bajo, que no permite implementar las diversas estrategias didácticas de los docentes, generando la desaprobación y el rechazo al área curricular. 
El problema formulado fue: ¿Cómo el software GeoGebra mejora el aprendizaje de la matemática en los estudiantes del nivel secundaria?

El objetivo de la presente investigación fue realizar un resumen sobre el uso de software GeoGebra como estrategia didáctica en matemática de estudiantes del nivel secundaria.

\section{MATERIALES Y MÉTODOS}

La presente investigación se desarrolló por medio de un estudio descriptivo de análisis documental, en torno a las publicaciones en software Geogebra, con la finalidad de poder describir la influencia del uso software Geogebra en al aprendizaje de matemática. La muestra estuvo constituida por $2 \mathrm{I}$ artículos de trabajos publicados en los últimos 5 años en revistas idexadas de las bases de datos de Google Académico y Scopus en idioma español e inglés. Los criterios de inclusión de los materiales incluyeron: artículos resultados de investigación e indexación de Google Académico y Scopus, artículos de tipo de investigación cuantitativa y el diseño cuasi experimental y no experimental. Se ha utilizado métodos de búsqueda exhaustiva como "Software Geogebra". Los indicadores calculados fueron: Temática trabajada, año de publicación, base de datos, tipo de estudio y diseño. El material documental recolectado se tabuló y diseñado para esta investigación y los resultados se presentan en tablas.

\section{RESULTADOS}

A continuación, se presentan a nivel descriptivo los principales indicadores empleados para dar cumplimiento al objetivo del estudio. Google Académico es la base de datos que registra un mayor número de publicaciones en los últimos 5 años; español es el idioma de mayor difusión del conocimiento en torno al uso de software Geogebra, seguido del inglés, respectivamente. los resultados descriptivos de los estudios seleccionados $(n=2 I)$ que abordan la mejora del rendimiento en matemática del nivel secundaria mediante el uso del software GeoGebra. Los resultados obtenidos una vez realizada la revisión para realizar el resumen respectivo aplicando los criterios de búsqueda descritos y analizando la asociación entre el uso del software GeoGebra y el rendimiento académico en matemática de estudiantes del nivel secundaria. Para la extracción de la información en la revisión se siguió el siguiente procedimiento: I) Autorías y año de publicación; 2) Diseño metodológico de estudio; 3) Variables de estudio; 4) Conclusiones.

\section{DISCUSIÓN}

La mayoría de estudios que abordan el aprendizaje en matemática a través de las puntuaciones obtenidas en pruebas revelan como el uso de software Geogebra permite la mejora del mismo. 
Varias autorías establecen que el uso del software Geogebra no solo podría mejorar el aprendizaje en matemática de forma directa, sino que también ayudaría a mejorar las habilidades del pensamiento crítico, el aprendizaje cooperativo y crea un ambiente flexible involucrando a los estudiantes. El aprendizaje en matemática como proceso consiste en conectar nodos o base de datos y puede estar fuera del ser humano en dispositivos o software como el GeoGebra, entendido como conocimiento aplicable en concordancia como lo afirma Siemens (citado por Cueva et al., 2019).

Los estudios realizados demuestran la necesidad de que el uso de software matemático GeoGebra integrado en forma inteligente y con creatividad contribuye a la mejora del rendimiento académico en matemática y en todas las ciencias. Zuña et al. (2020) sostienen que las plataformas virtuales son idóneas para fomentar la colaboración en el contexto educativo y realizan también una reflexión sobre las contribuciones en los procesos de enseñanza y Morales (2020) afirma que los actores educativos, principalmente docentes y estudiantes, se apropian de las TIC desde distintos lugares, que reconfiguran en distintos niveles y dimensiones de su vida escolar, los procesos de enseñanza y aprendizaje, e incluso su identidad.

Arévalo y Gonzáles (2020) afirman que la implementación de técnicas de enseñanza con ayuda de herramientas informáticas es usado para facilitar la enseñanza de integrales, métodos numéricos, procedimientos matemáticos y Salas (2020) sostiene que la convergencia entre estudiantes y docentes debe generar soluciones creativas a problemas reales haciendo buen uso de la tecnología y en ese sentido se busca que los inmigrantes digitales que son los docentes deben adquirir las habilidades de los nativos digitales para poder interactuar con ellos y lograr los aprendizajes significativos. Manzo et al. (2020) sostienen que las herramientas para la práctica docente como pizarras interactivas, aulas virtuales y otros recursos digitales disponibles en la actualidad son muy buenas para la práctica docente, lo cual contribuye en la eficacia de la tecnología digital aplicada en clases con los estudiantes y en consecuencia mejora de la enseñanza y aprendizaje. Resulta esencial destacar las principales limitaciones de esta revisión. En primer lugar, puede destacarse el rango de búsqueda. Los estudios fueron acotados sobre los últimos cinco años con el fin de obtener una visión actualizada del tema investigado.

\section{REFERENCIAS}

Álvarez - Melgarejo, C., Cordero - Torres, J., González Bareño, J., \& SepúlvedaDelgado, O. (2019). Software GeoGebra como herramienta en enseñanza y aprendizaje de la Geometría. EDUCACIÓN Y CIENCIA, (22), 387-402. Recuperado a partir de https://revistas.uptc.edu.co/index.php/educacion_y_ciencia/article/view/I005 9. 
Arévalo, B. y Gonzáles, A. (2020). Enseñanza de integrales mediante métodos numéricos por medio del uso de las TIC. Sinergias educativas. Octubre - Diciembre Vol. 5 42020 http://sinergiaseducativas.mx/index.php/revista/ elSSN: 266I-666I Pag 2I34.

Arteaga Valdés, E., Medina Mendieta, J. F., \& Del Sol Martínez, J. L. (2019). El GeoGebra: una herramienta tecnológica para aprender matemática en la Secundaria Básica haciendo matemática. Revista Conrado, I5(70), 102-108. Recuperado de http://conrado.ucf.edu.cu/index.php/conrado.

Barros Bastidas, C., \& Turpo Gebera, O. (20/8). Factors influencing the scientific production of university professors: a systematic review. Factores Que Influyen En La Producción Científica de Los Docentes Universitarios: Una Revisión Sistemática., I I (22), 225-234. http:// 0.0.85.43/pensam.vI Ii2 II.276\%0Ahttps://ezproxy.uniandes.edu.co:8443/login?url=http://search.ebscohost.c om/login.aspx?direct=true\&db=fua\&AN=|4| 223533\&lang=es\&site=ehost-live

Bolaños, Cynthia; Ruiz-Hidalgo, Juan Francisco (20।8). Demostrando con GeoGebra. Números. Revista de Didáctica de las Matemáticas, 99, Pp. I53-17I.

Cueva, J., García, A., \& Martínez, O. (2019). El conectivismo y las TIC: Un paradigma que impacta el proceso enseñanza aprendizaje. Revista Scientific, 4(I4), 205-227, eISSN: 2542-2987. Recuperado de: https://doi.org/10.29394/Scientific.issn.25422987.2019.4.14.10.205-227.

Díaz, Stephanie; Gutiérrez, Rafael Enrique; Luque, Rafael E. (2018). Propuesta didáctica para abordar el tema de la función trigonométrica $f(x)=\tan (\boldsymbol{x})$ con el software GeoGebra. En Afonso, Hugo; Bruno, Alicia; de la Coba, Dolores; Domínguez, Miguel; Duque, Yanira; García, Fátima; Plasencia, Inés (Eds.), Números, Revista de Didáctica de las Matemáticas Números, 97. (pp. 83-9l). La Laguna (Tenerife), España: Sociedad Canaria Isaac Newton de Profesores de Matemáticas.

Diaz-Nunja, Luis, Rodríguez-Sosa, Jorge, \& Lingán, Susana K. (20l8). Enseñanza de la geometría con el software GeoGebra en estudiantes secundarios de una institución educativa en Lima. Propósitos y Representaciones, 6(2), 217234. https://dx.doi.org/I0.205II/pyr20I8.v6n2.25I.

Filiz Tuba Dikkartin Övez (2018). The Impact of Instructing Quadratic Functions with the Use of

Geogebra Software on Students' Achievement and Level of Reaching Acquisitions. International Education Studies; Vol. II, No. 7; 2018 ISSN I9|3-9020 E-ISSN I 9139039.

Guachún, Freddy; Mora, Blanca (2019). El software GeoGebra como recurso para la enseñanza de la función lineal: Una propuesta didáctica. Números. Revista de Didáctica de las Matemáticas, I0I, PP. 103-II2. 
Jiménez García, J. G., \& Jiménez Izquierdo, S. (2017). GeoGebra, una propuesta para innovar el proceso enseñanza-aprendizaje en matemáticas. Revista Electrónica Sobre Tecnología, Educación Y Sociedad, 4(7). Recuperado a partir de https://www.ctes.org.mx/index.php/ctes/article/view/654.

Lissette Rodríguez Rivero, Teresita Linares Rodríguez, Jorge Bello Brito, Ana Teresa Garriga González (2018). Experiencias del uso de GeoGebra en la Esbu "Ramón Leocadio Bonachea Hernández". III Conferencia Científica Internacional UCIENCIA 2018 Universidad de las Ciencias Informáticas. La Habana, Cuba.

M. K. Tay, and T. Mensah-Wonkyi (20l8). Effect of using Geogebra on senior high school students' performance in circle theorems. African Journal of Educational Studies in Mathematics and Sciences Vol. I4.

Manzo, C., Chonillo, F. y Sylva, L. (2020). Sinergias educativas. Enero - junio Vol. 5 - I 2020 http://sinergiaseducativas.mx/index.php/revista/elSSN: 2662-666 I

Mendoza, J., Insuasti, R. y Baquero, J. (2018): "Software GeoGebra como herramienta didáctica para el aprendizaje de las ciencias exactas un caso de estudio", Revista Atlante: Cuadernos de Educación y Desarrollo (mayo 2018). En línea: https://www.eumed.net/rev/atlante/2018/05/software-geogebra.html.

Morales, M. (2020). Apropiación cultural: una perspectiva para analizar la incorporación de tic en la práctica docente. Sinergias educativas. Octubre - Diciembre Vol. 5 42020 http://sinergiaseducativas.mx/index.php/revista/ elSSN: 266I-666I Pag 7I84

Munandar, Usman, Saminan (2020). Analisys of the impact of mathematical learning with geogebra asistance on critical thinking ability. The 6th Annual International Seminar on Trends in Science and Science Education.

Nurhayati et al. (2020). Implementation of software geogebra on triangles. Journal of Physics: Conference Series I569 042068.

Ramírez, Mayerlin; Vargas, Leonardo; Vásquez, Fernando (2018). Desarrollo del pensamiento aleatorio en el proceso de aprendizaje de las medidas de tendencia central mediante GeoGebra en estudiantes de noveno grado. En Valbuena, Sonia; Vargas, Leonardo; Berrío, Jesús (Eds.), Encuentro de Investigación en Educación Matemática (pp. 37I-379). Puerto Colombia, Colombia: Universidad del Atlántico.

Rimarachi, F. (2019). Uso del geogebra en el aprendizaje de resolución de problemas de ecuaciones cuadráticas en educación secundaria. Revista de Ciencias Naturales (julio 2019). http://repositorio.unap.edu.pe/handle/UNAP/I4I4I.

Salas, M. (2020). Convergencia entre Nativos Digitales e Inmigrantes Digitales. Sinergias educativas

Enero - junio Vol. 5 -I - 2020 http://sinergiaseducativas.mx/index.php/revista/elSSN: 2662-666I

Sarmiento-Espinoza, W., \& Luna-Altamirano, K. (2017). Aplicación del software GeoGebra en prácticas matemáticas bajo una metodología constructivista. Killkana Social, I(2), 45-50. https://doi.org/l0.2687I/killkana_social.vli2.38. 
Sarabia, A. (1995). La Teoría General de Sistemas. (Primera edición). Madrid - España. Ueno, Carlos (2016). Demostraciones geométricas automáticas en GeoGebra. En Afonso, Hugo; Bruno, Alicia; de la Coba, Dolores; Domínguez, Miguel; Duque, Yanira; García, Fátima; Noda, Ma Aurelia; Plasencia, Inés (Eds.), Números, Revista de Didáctica de las Matemáticas Números, 93 . (pp. I4I-I50). La Laguna (Tenerife), España: Sociedad Canaria Isaac Newton de Profesores de Matemáticas.

YA Wassie, GA Zergaw (20I8). Capabilities and Contributions of the Dynamic Math Software, GeoGebra---A Review North American GeoGebra Journal Volume 7, Number I, ISSN 2162-3856.

Y Selvy et al. (2020). Improving students' mathematical creative thinking and motivation through GeoGebra assisted problem based learning. Journal of Physics: Conference Series I460 0I 2004.

Zenteno Ruiz, Flaviano Armando, Rivera Espinoza, Tito Armando, \& Pariona Cervantes, Daniel Joel. (2020). Tratamiento de las medidas de dispersión por medio del software geogebra. Revista Universidad y Sociedad, I2(I), 244-250. Epub 02 de febrero de 2020. Recuperado en 20 de diciembre de 2020, de http://scielo.sld.cu/scielo.php?script=sci_arttext\&pid=S22 I 8 $36202020000100244 \& \operatorname{lng}=e s \&$ tlng $=p t$.

Zulnaidi, H. et al. (2020). Efecto del uso de GeoGebra en el rendimiento de los estudiantes de matemáticas de secundaria. Tecnologías de la información y la educación Volumen 25, Número I, I de enero de 2020, Páginas 5I-72.

Zuña, E., Romer, W., Palma, J. y Soledispa, C. (2020). Plataformas virtuales y fomento del aprendizaje colaborativo en estudiantes de Educación Superior. Sinergias Educativas Enero - $\quad$ Junio Vol. 5 - $\quad 1 \quad 2020$ http://sinergiaseducativas.mx/index.php/revista/elSSN: 2662-666I DOI: https://doi.org//0.37954/se.v5il.7I. 